\title{
Silencing of CXCR4 by RNA interference inhibits cell growth and metastasis in human renal cancer cells
}

\author{
LINHUI WANG ${ }^{*}$, TING HUANG ${ }^{*}$, WEI CHEN ${ }^{*}$, XIAOFENG GAO, TIE ZHOU, ZHENJIE WU and YINGHAO SUN \\ Department of Urology, Changhai Hospital, Second Military Medical University, Shanghai 200433, P.R. China
}

Received April 29, 2012; Accepted July 5, 2012

DOI: $10.3892 /$ or.2012.2028

\begin{abstract}
Renal cell carcinoma (RCC) is the third most common genitourinary malignancy, accounting for $3 \%$ of cancer in adults. The mortality and morbidity of RCC is strongly associated with its high propensity to metastasize to specific organs. This may be attributed to the fact that the CXCR4 G protein-coupled receptor (GPCR) on RCC cells mediates chemoattraction toward stromal-derived factor 1 (SDF-1) secreted by target organs. RNA interference (RNAi), which has been proven to be a powerful tool for suppressing gene expression, may lead to novel strategies for treating RCC. Our previous experiments confirmed that RCC A-498 cells overexpressing CXCR4 are associated with increased invasiveness. In this study, we constructed recombinant CXCR4-RNAi plasmids and transfected them into A-498 cells in vitro. Reverse transcription polymerase chain reaction (RT-PCR) and western blotting revealed that CXCR4 was downregulated in transfected cells compared with control cells. Our results from MTT and transwell migration assays indicated that specific downregulation of CXCR4 inhibited cell growth, invasiveness and migration. Flow cytometric analysis indicated that silencing of CXCR4 in A-498 cells by RNA interference induced cell apoptosis in RCC in vitro. Thus, siRNA targeting of CXCR4 can effectively inhibit the growth and metastasis of RCC cells and may be a promising innovative anticancer therapy.
\end{abstract}

\section{Introduction}

Metastatic renal cell carcinoma (RCC) is associated with a poor prognosis, with a median survival of 8 months (1). At the time RCC are identified, in most cases the tumors have already progressed and are generally resistant to chemo-

Correspondence to: Professor Yinghao Sun, Department of Urology, Changhai Hospital, Second Military Medical University, 168 Changhai Road, Shanghai 200433, P.R. China

E-mail: sunyh@medmail.com.cn

${ }^{*}$ Contributed equally

Key words: CXCR4, RNA interference, renal cell carcinoma therapy and radiotherapy. Although some patients respond to immunotherapy, many cases are resistant to interleukin-2 or interferon- $\alpha$ (IFN- $\alpha$ ) therapy and the overall response rate is less than $20 \%$ (2). The factors leading to this lack of response remain under investigation. Therefore, it is necessary to find more effective, universal treatments for this disease. Molecular targeting of abnormal signal transduction pathways is promising for the treatment of many diseases. Many studies have suggested that chemokine played a significant role, not only in immune and inflammatory responses, but also in malignant cell growth and progression $(3,4)$. The CXCR4 chemokine receptor belongs to the group of seven transmembrane $G$ protein-coupled receptors (GPCRs) which are ubiquitously expressed in various normal cells and tissues, including neurons, lymphatic tissues, microglia and hematopoietic cells $(3,5)$. CXCR4 and its ligand, stromal cell-derived factor-1 (SDF-1), currently termed CXCL12, are believed to play significant roles in tumorigenesis and metastasis (6). Based on these studies, downregulation of CXCR4 may provide a therapeutic strategy for inhibiting tumors metastasis and for enhancing the survival of patients with RCC. AMD3100 is a specific CXCR4 antagonist that is used in the clinic to improve the success of stem cell transplantation in cancer patients (7). However, drug resistance may limit the use of AMD3100 in clinical applications (8). In another clinical study, two patients treated with 40 and $160 \mathrm{mg} / \mathrm{kg} / \mathrm{h}$ AMD3100 had unexpected premature ventricular contractions, which resulted in the discontinuation of the drug (9). Surprisingly, AMD3100 reduced growth of lymphomas in vivo when administered three times weekly, but enhanced tumor growth after continuous drug infusions $(6,10)$. However, recently the advent of RNAi-directed 'knock-down' has sparked a revolution in somatic cell genetics, allowing for inexpensive and rapid analysis of gene function in mammals, and might be exploited for gene therapy in the future (11).

Our previous experiment has confirmed that the RCC A-498 cell line, which expresses high level of CXCR4, was associated with increased invasiveness (12). In the present study, we employed RNAi to inhibit CXCR4 expression in A-498 cells and investigated the effect of CXCR4 suppression on the proliferation and apoptosis of those cells in vitro.

\section{Materials and methods}

Cell line and culture. A-498 cells (ATCC, Manassas, VA, USA), a human renal cell carcinoma cell line, were grown 
at $37^{\circ} \mathrm{C}$ in a humidified atmosphere of $95 \%$ air and $5 \% \mathrm{CO}_{2}$ in ATCC-formulated Eagle's minimum essential medium (EMEM, cat. no. 30-2003, ATCC) supplemented with $10 \%$ fetal bovine serum (Hyclone Corp., USA).

Construction of the CXCR4 specific short hairpin RNA (shRNA) expression vector and stable transfection. Double chains of oligonucleotide with complementary sequences that can code short hairpin RNA (shRNA) were obtained from GenePharma Co., Ltd. (Shanghai, China). The siRNA recognized nucleotides 525-543 of CXCR4 mRNA (GAAGCAT GACGGACAAGTA) of the transcript according to the NCBI database (GeneID7852). CXCR4-shDNA oligos are listed below: 5'-CACCGAAGCATGACGGACAAGTATTCAAGA GATACTTGTCCGTCATGCTTCTTTTTTTG-3' (sense) and 5'-GATCCAAAAAAGAAGCATGACGGACAAGTATCTC TTGAATACTTGTCCGTCATGCTTC-3' (antisense). The target sequence of the negative control group named shRNAcontrol was 5'-GTTCTCCGAACGTGTCACGT-3' (sense) and 5'-ACGTGACACGTTCGGAGAAT-3' (antisense), which has no homology with that of human or mice. The hairpin loop region was annealed with its complementary strand and was cloned into the pGPU6/GFP/Neo plasmid vector (GenePharma Co., Ltd.) carrying a green fluorescent protein (GFP) reporter gene and genes for ampicillin and neomycin resistance. The shRNA expression vectors were transfected into A-498 cells using the Lipofectamine 2000 transfection reagent (Invitrogen, Carlsbad, CA, USA) according to the manufacturer's instructions. G418 was added into the culture medium $(500 \mathrm{mg} / \mathrm{ml}$, Gibco Bio-Cult) after $48 \mathrm{~h}$. Stable G418-resistant clones were obtained after 4 weeks. The expanded cells were then used for subsequent studies.

$R N A$ isolation and reverse transcription polymerase chain reaction (PCR). Total RNA was isolated from cells by using TRIzol Reagent (Invitrogen). cDNA synthesis was performed using reverse transcription reagents (Promega, Madison, WI, USA). Real-time PCR was performed using SYBR-Green I Mix (ABI, Foster City, CA, USA) and an ABI Prism 7700 Sequence Detection system (ABI) according to the manufacturer's instructions. The primer sequences for the genes and expected product sizes were as follows: 5'-GGAAAAGAGG GGAGGAGAG-3' (sense) and 5'-CACTTCCAATTCAG CAAGCA-3' (antisense) for CXCR4, 5'-ACCACCATGAGA AGGCTGG-3' (sense) and 5'-CTCAGTGTAGCCCAGGA TGC-3' (antisense) for GAPDH.

Western blotting. Stable transfected A-498 cells were lysed with a denaturing SDS-PAGE sample buffer using standard methods, and the resulting lysates were cleared by centrifugation. Proteins were transferred onto a nitrocellulose membrane, blocked with Tris-buffered saline plus $0.1 \%$ Tween-20 (TTBS) containing 5\% non-fat milk for $2 \mathrm{~h}$. The membrane was incubated overnight with the rabbit anti-human primary polyclonal antibody against CXCR4 (Abcam, USA) at 1:1000 dilution at $4^{\circ} \mathrm{C}$, then washed and incubated with the HRP-conjugated goat anti-rabbit IgG (Pierce, Rockford, IL, USA) at $1: 2000$ for $1 \mathrm{~h}$ at $37.0^{\circ} \mathrm{C}$. Actin was probed using a rabbit monoclonal antibody against human protein and an anti-rabbit IgG as secondary antibody
(Santa Cruz Biotechnology). Finally, the bands were visualized by chemiluminescence using a chemiluminescence kit (Invitrogen) and the specific bands were recorded on X-ray film. BandScan 5.0 software was used for gray scale scan to evaluate the relative value of protein expression.

Cell proliferation assay. Cells in the log-growth phase were harvested, suspended at a density of $\sim 1 \times 10^{4}$ cells/well. After $24 \mathrm{~h}$ of culture, $20 \mu \mathrm{l}$ MTT $(5 \mathrm{mg} / \mathrm{ml}$, Sigma) was added to each well. The plates were then incubated at $37^{\circ} \mathrm{C}$ for $4 \mathrm{~h}$. Then culture medium was removed and $150 \mu 1$ of DMSO (Sigma) was added and thoroughly mixed for $10 \mathrm{~min}$. Absorbance of each well was measured at a wavelength of $490 \mathrm{~nm}$ and the numbers of surviving cells were calculated.

Invasion and migration assay. The invasion assay was performed using an $8 \mu \mathrm{m}$ pore size transwell chamber in 24-well plates (Corning Costar, Cambridge, MA, USA). A-498 cells $\left(1.0 \times 10^{5}\right)$ in $500 \mu$ l of serum-free MEM medium were loaded into the top chamber with fetal bovine serum placed in the bottom chamber as a chemoattractant. After further incubation at $37^{\circ} \mathrm{C}$ for $10 \mathrm{~h}$, the cells on the top of the filters were removed with cotton swabs. The cells on the lower surface of the filters were fixed in $4 \%$ paraformaldehyde and stained with $0.1 \%$ crystal violet. The crystal violet was removed and the cells were washed three times with PBS, and then the remaining crystal violet that had stained the migrated cells was eluted with one wash with $33 \%$ acetic acid. The OD540 nm of the eluted crystal violet was determined as a measure of migrated cells. The cell migration assay was performed in a similar mode, except that cells were seeded into the uncoated filter and incubated for $24 \mathrm{~h}$.

Analysis of apoptosis by flow cytometry. Apoptosis was determined with an Annexin V-FITC/propidium iodide Apoptosis Detection kit (Bipec Biopharma Corp., Cambridge, MA) according to the manufacturer's protocol. The cells were washed with PBS and subsequently incubated for $5 \mathrm{~min}$ at room temperature in the dark in $500 \mu \mathrm{l}$ of $1 \mathrm{X}$ binding buffer containing $5 \mu \mathrm{l}$ of Annexin V-FITC and $10 \mu \mathrm{l}$ of propidium iodide. Afterwards, apoptosis was analyzed by flow cytometry (FCM).

Statistical analysis. All measurements were carried out with the same instrument under the same experimental condition. Data are expressed as means \pm standard deviation (SD). All data were analyzed statistically by one-way analysis of variance (ANOVA) and $\mathrm{P}<0.05$ was considered significant.

\section{Results}

Assessment of the effect of CXCR4 siRNA in A-498 cells. After the plasmid vector with green fluorescent protein (GFP) was transfected into the cells using Lipofectamine 2000 transfection reagent, it produced a green fluorescent wave. Microscopic analysis revealed that CXCR4 RNAi induced morphological changes in A- 498 cells characterized by irregular morphology, less dense structure and were smaller in volume compared with untransfected cells (Fig. 1A). The fluorescent microphoto indicated that transfection was successful (Fig. 1B). 
A

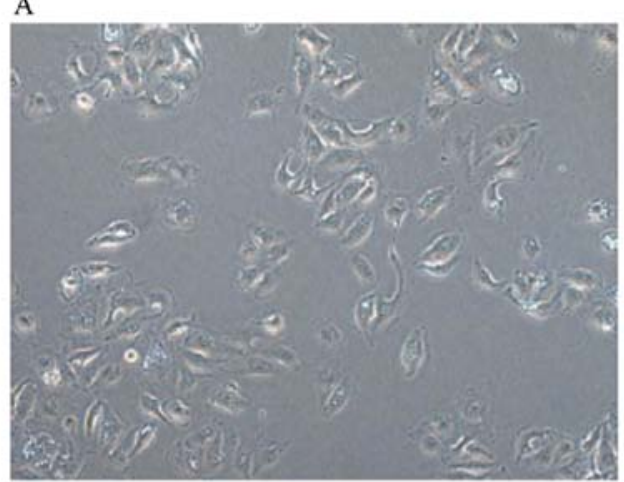

B

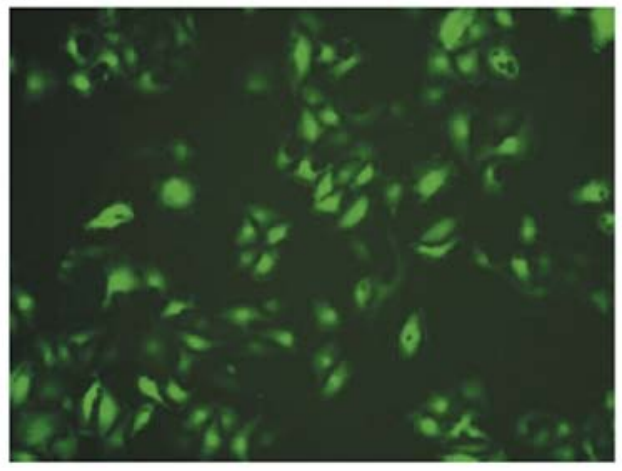

Figure 1. Light and fluorescent micrographs of cells transfected using plasmid vector expressing green fluorescent protein (magnification, x200). (A) Light microscopy image; (B) fluorescent microscopy image. High intensity of fluorescence indicated a high efficiency of transfection.

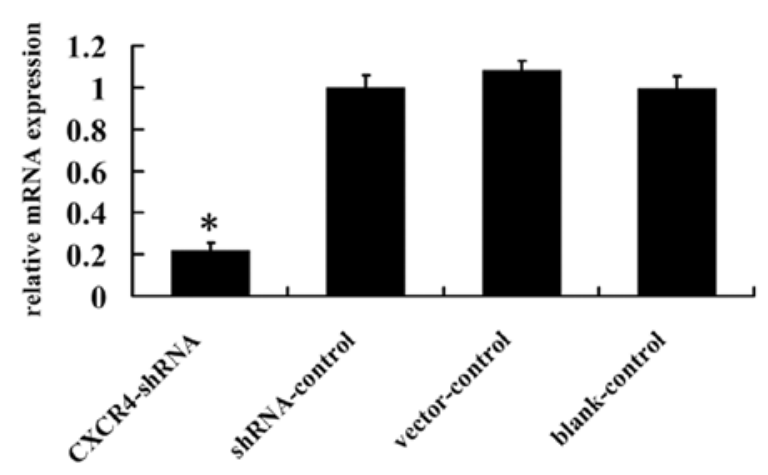

Figure 2. Expression of CXCR4 mRNA in A-498 cells. The graph represents data from 6 independent experiments illustrating that CXCR4-shRNA reduces CXCR4 mRNA in A-498 cells compared to shRNA-control, vectorcontrol, or blank-control. "Significant difference from control groups, $\mathrm{P}<0.01$.

RNAi inhibits CXCR4 mRNA expression in A-498 cells. To study the silencing effect of CXCR4 shRNA, CXCR4 expression was evaluated by RT-PCR. As shown in Fig. 2, CXCR4 mRNA expression in CXCR4-shRNA transfected A-498 cells was reduced $78.9 \%$, compared to shRNA-control, vector-control and blank-control cells $(\mathrm{P}<0.01)$, indicating that the corresponding mRNA sequence for CXCR4 RNAi was specific to the intended target.

RNAi inhibits CXCR4 protein expression in A-498 cells. CXCR4-shRNA resulted in a significant decrease in CXCR4

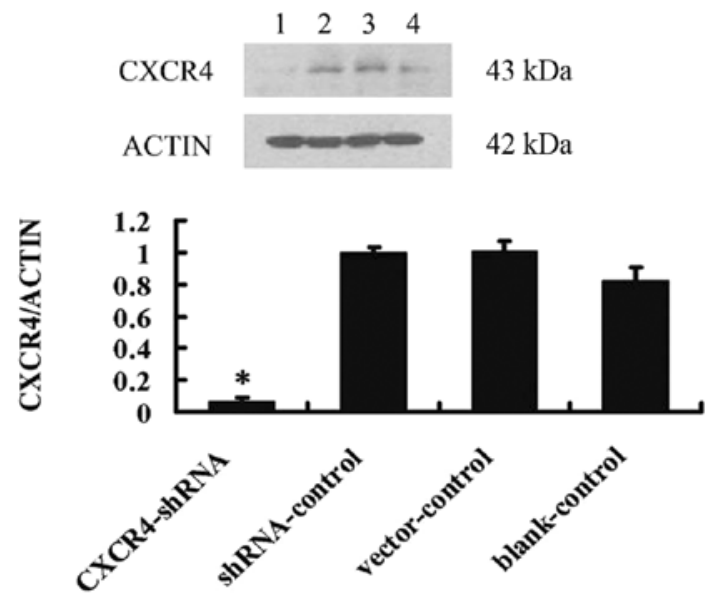

Figure 3. Expression of CXCR4 protein in A-498 cells by western blot analysis. The image is from 5 independent experiments, which illustrates that CXCR4-shRNA reduces CXCR4 mRNA in A-498 cells compared to shRNA-control, vector-control, or blank-control. Lane 1, CXCR4-shRNA cells; lane 2, shRNA-control cells; lane 3, vector-control cells; lane 4, blankcontrol cells. *Significant difference from control groups, $\mathrm{P}<0.01$.

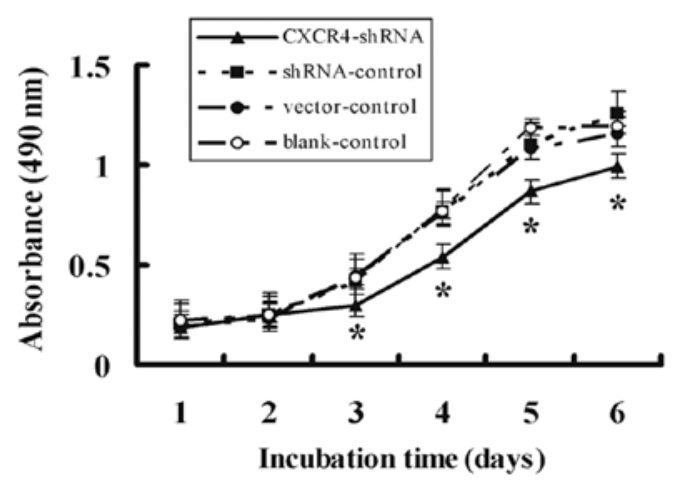

Figure 4. Cell proliferation detected by MTT. Cell proliferation was inhibited notably in a time-dependent manner. The data at each time point was averaged values from 6 replicates. *Significant difference from control groups on the same day, $\mathrm{P}<0.01$.

protein levels $(11.67 \pm 3.09 \%$ normalized to $\beta$-actin) (Fig. 3) compared with shRNA-control cells $(55.50 \pm 3.72 \%$ normalized to $\beta$-actin), vector-control $(53.44 \pm 5.65 \%$ normalized to $\beta$-actin) and blank-control cells $(50.63 \pm 8.17 \%$ normalized to $\beta$-actin) $(\mathrm{P}<0.01)$, suggesting that $\mathrm{CXCR} 4-$ shRNA strongly inhibits CXCR4 expression.

CXCR4 shRNA inhibits A-498 cell proliferation. The growth rate of cells after transfection was examined using MTT assay for 6 days. As shown in Fig. 4, CXCR4-shRNA reduced the growth of A-498 cells significantly as compared to shRNA, vector, and blank-controls $(\mathrm{P}<0.01)$. Cell proliferation was inhibited notably in a time-dependent manner for CXCR4shRNA cells and the highest inhibitory rate was $31.33 \pm 1.78 \%$ on Day 3. There was no difference in inhibition among shRNA, empty vector, or blank-controls $(\mathrm{P}>0.05)$.

CXCR4 shRNA inhibits A-498 cell invasion and migration. We evaluated whether suppression of CXCR4 altered 


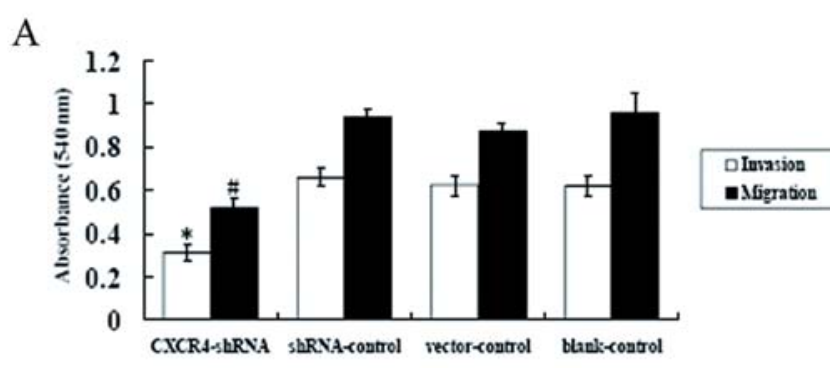

B
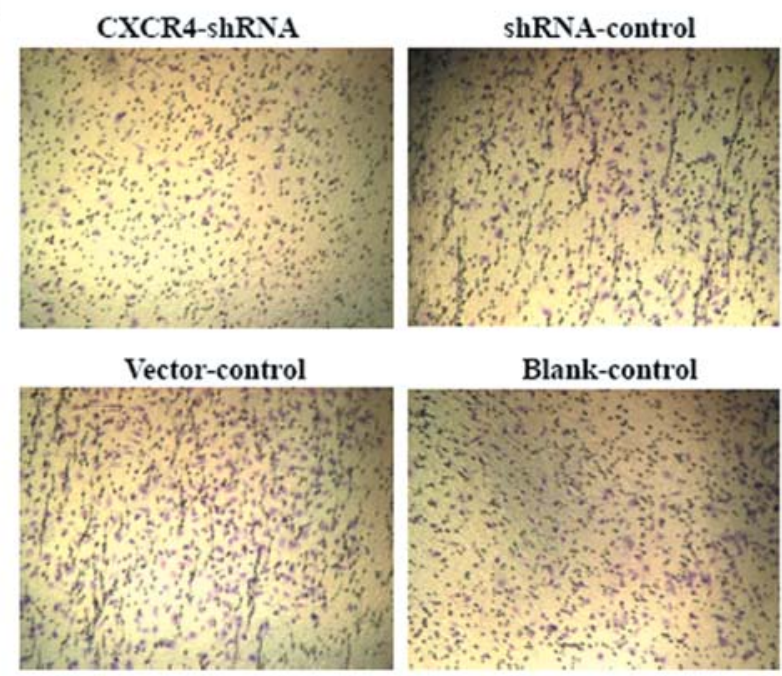

Figure 5. Invasion and migration assay. (A) Compared with the shRNAcontrol, vector-control, and blank-control cells, the cell invasiveness and migration of CXCR4-shRNA cells were reduced. (B) The polycarbonate filters were stained with crystal violet and viewed with a light microscope (magnification, x100). These experiments were performed 6 times "Significant difference from control groups in white bars, $\mathrm{P}<0.01$; "Significant difference from control groups in black bars, $\mathrm{P}<0.01$.

Table I. Effect of CXCR4 gene depletion on A-498 cell apoptosis.

\begin{tabular}{lccc}
\hline & \multicolumn{3}{c}{ Apoptosis rate (\%) } \\
\cline { 2 - 4 } Groups & Early phase & Late phase & Total \\
\hline Negative-control & $3.65 \pm 0.21$ & $8.06 \pm 0.48$ & $11.72 \pm 0.47$ \\
Vector-control & $3.58 \pm 0.16$ & $7.41 \pm 0.34$ & $10.99 \pm 0.38$ \\
Blank-control & $4.53 \pm 0.32$ & $8.28 \pm 0.58$ & $12.83 \pm 0.69$ \\
CXCR4 shRNA & $17.74 \pm 0.31^{\text {a }}$ & $15.01 \pm 0.26^{\text {a }}$ & $32.76 \pm 0.38^{\text {a }}$ \\
\hline
\end{tabular}

${ }^{\mathrm{a}} \mathrm{P}<0.05$ vs. control groups; $\mathrm{n}=6$; mean $\pm \mathrm{SD}$.

the motility of A-498 cells across transwell polycarbonate membranes. As shown in Fig. 5A, compared with shRNA, vector, and blank controls, the cell invasiveness and migration of CXCR4-shRNA cells were reduced by $51.28 \pm 1.64 \%$ and $43.73 \pm 2.75 \%$, respectively $(\mathrm{P}<0.01)$. In contrast, there were no differences observed between shRNA, vector, and blank controls $(\mathrm{P}>0.05)$. Fewer CXCR4-shRNA-transfected cells than shRNA, vector, and blank control-tansfected cells were observed when the polycarbonate filters were stained with crystal violet (Fig. 5B). Our data suggested that silencing by CXCR4-shRNA can inhibit the invasive potential of A-498 cells.

Effect of CXCR4 shRNA on cell apoptosis. The cell apoptosis assay was measured by FCM. Apoptosis rate was $32.76 \pm 0.56 \%$ in CXCR4 shRNA cells, $11.72 \pm 0.70 \%$ in shRNA-control cells, $10.99 \pm 0.50 \%$ in vector-control cells, and $12.83 \pm 0.90 \%$ in blank-control cells. As shown in Table I, compared with control or untreated cells, more necrotic cells were detected in the cells treated with plasmid-mediated CXCR4 shRNA $(\mathrm{P}<0.01)$.

\section{Discussion}

Chemokines are 8 to $10-\mathrm{kDa}$ cytokines that are classified into four groups (CXC, $\mathrm{CC}, \mathrm{C}$ and $\mathrm{CX} 3 \mathrm{C}$ ) based on the position of the first two cysteines (13). CXCR4, a GPCR, is constitutively expressed in a wide range of normal tissues and its expression is enhanced in many solid tumors (14). Quantitative analysis of CXCR4 gene expression has been proposed as a prognostic marker and a predictor of potential metastasis in colorectal cancer (15), breast cancer (16), gastric cancer (17), acute myelogenous leukemia (18), osteosarcoma (19) and prostate cancer (20). Zagzag et al found that CXCR4 mRNA is upregulated in kidney cancer samples compared to adjacent normal tissue indicating a closely relation between CXCR4 and tumor cell dissemination and invasion (21). In addition, it was found that strong CXCR4 expression in renal cell carcinoma is associated with advanced T status (22). However, few reports have investigated the effects of siRNA directed inhibition of CXCR4 in RCC. Accordingly, to gain more insight into the effect of CXCR4 in A-498 cells, we selectively knocked down CXCR4 expression using RNAi and observed the subsequent effects in A-498 cells.

In this study, we successfully constructed a plasmid expression vector that contained a CXCR4 short hairpin RNA expression cassette. Our study showed that in stable transfected A-498 cells, the shRNA was introduced into the cytoplasm, forming an RNA-induced silencing complex with other nucleus enzymes. We found that CXCR4 mRNA and protein production were downregulated. In contrast, CXCR4 expression was unchanged in the control groups, which confirmed that high specific gene suppression by RNAi was achieved.

We previously reported that overexpression of CXCR4 in A-498 cells is associated with increased Matrigel matrix invasion (12). In this study we observed that RNAi-mediated silencing of CXCR4 decreased cell proliferation by MTT and Matrigel invasion assays. Our results suggest that CXCR4 is a positive regulator in the growth of A-498 cells and thus supports A-498 cell proliferation. Previous studies have found that CXCR4 nuclear localization may be responsible for these metastatic changes $(12,23)$. The nuclear localization of CXCR4 is comparable to the mechanism exhibited by the epidermal growth factor receptor (EGFR), another membrane receptor, which translocates to the nucleus after binding to epidermal growth factor, and increases the promoter region of the cyclin D1 gene transcription (24). Cyclin D1 is a regulatory kinase critical for progression through the G1 to $\mathrm{S}$ phase transition of the cell cycle and is involved in cancer cell growth (25), 
and thus nuclear translocation of EGFR leads to increased cell proliferation.

Increased resistance to apoptosis is a common characteristic of cancer cells, and alterations in the apoptotic pathway contribute to tumorigenesis and chemotherapy resistance in carcinoma cells. In this study, we also observed that A-498 RCC cells treated with CXCR4 shRNA had an increased apoptotic rate. Previous studies indicated that suppression of CXCR4 reduces the induction of apoptosis in other types of cancer cells such as neuroectodermal tumors (26), antagonists medulloblastoma (27), and breast cancer cell lines in vitro (28). Furthermore, another report showed that crosstalk between CXCR4 and integrin signaling increased adhesion of small cell lung cancer (SCLC) cells to stromal cells, which protected these cells from chemotherapy-induced apoptosis conferring chemoresistance (29). CXCR4 blockade reduced adhesion and survival signals from the tumor microenvironment to SCLC cells and increased sensitivity to chemotherapy induced apoptosis (30). As an anti-apoptotic factor, Bcl-2 protects cells from apoptosis by regulating mitochondria membrane potential and preventing mitochondrial cytochrome c release (31). Carmen et al reported that Bcl-2 activity decreases after addition of CXCR4 antagonists, leading to A-498 cell death through a mitochondrial apoptotic pathway (32). After binding to CXCR4, SDF-1 causes mobilization of calcium, decrease of cyclic AMP within the cells, and activates multiple signal transduction pathways such as PI3K/Akt/eNOS pathway, which can enhance cell proliferation, migration, survival, and angiogenesis signals by inducing eNOS activity (33). It has also been reported that a number of Akt downstream target molecules such as nitric oxide (34), and Bcl-2 family members including Bcl-xL, $\mathrm{Bcl}-\mathrm{w}, \mathrm{Bad}$ and $\mathrm{Bax}$ (35) are involved in regulating tumor cell apoptosis (36). In summary, these findings provide evidence that downregulation of CXCR4 expression has an apoptotic effect in A-498 cells and this mechanism could lead to an anti-tumor effect.

In conclusion, our present study shows that silencing of CXCR4 using RNA interference could effectively inhibit proliferation, invasion and metastasis in A-498 RCC cells. Traditionally, the standard treatment for patients with RCC is surgical resection in the case of localized disease (37). However, for metastatic RCC, gene therapy based on RNA interference-mediated silencing of CXCR4 might be a promising and innovative anticancer therapy. In addition, more studies are required to assess the pharmacokinetics and improve the tissue specific targeting of certain genes with siRNA.

\section{Acknowledgements}

This study was supported by the Science and Technology key project of basic research of Shanghai, China (no. 10JC1417800).

\section{References}

1. Godley P and Kim SW: Renal cell carcinoma. Curr Opin Oncol 14: 280-285, 2002.

2. Fishman $M$ and Seigne J: Immunotherapy of metastatic renal cell cancer. Cancer Control 9: 293-304, 2002.

3. Vicari AP and Caux C: Chemokines in cancer. Cytokine Growth Factor Rev 13: 143-154, 2002.
4. Navarini-Meury AA and Conrad CC: Melanoma and innate immunity-active inflammation or just erroneous attraction? Melanoma as the source of leukocyte-attracting chemokines. Semin Cancer Biol 19: 84-91, 2009.

5. Skommer J, Wlodkowic D and Pelkonen J: CXCR4 expression during tumour cell death. Leuk Res 31: 1155-1156, 2007.

6. Burger JA and Kipps TJ: CXCR4: a key receptor in the crosstalk between tumor cells and their microenvironment. Blood 107: 1761-1767, 2006.

7. Broxmeyer HE, Orschell CM and Clapp DW: Rapid mobilization of murine and human hematopoietic stem and progenitor cells with AMD3100, a CXCR4 antagonist. J Exp Med 201: 1293-1305, 2005.

8. Labrosse B, Brelot A and Heveker N: Determinants for sensitivity of human immunodeficiency virus coreceptor CXCR4 to the bicyclam AMD3100. J Virol 72: 6381-6388, 1998.

9. Hendrix CW, Collier AC, Lederman MM, et al: Safety, pharmacokinetics, and antiviral activity of AMD3100, a selective CXCR4 receptor inhibitor, in HIV-1 infection. J Acquir Immune Defic Syndr 37: 1253-1262, 2004.

10. Paul S, Mancuso P, Rabascio C, et al: In Vitro and Preclinical Activity of the Novel AMD3100 CXCR4 Antagonist in Lymphoma Models. In: American Society of Hematology Annual Meeting, 2002; Philadelphia, PA, USA: Blood, 2002.

11. Duxbury MS and Whang EE: RNA interference: a practical approach. J Surg Res 117: 339-344, 2004.

12. Wang L, Wang Z, Yang B, et al: CXCR4 nuclear localization follows binding of its ligand SDF-1 and occurs in metastatic but not primary renal cell carcinoma. Oncol Rep 22: 1333-1339, 2009.

13. Zlotnik A and Yoshie O: Chemokines: a new classification system and their role in immunity. Immunity 12: 121-127, 2000.

14. Zlotnik A: Chemokines and cancer. Int J Cancer 119: 2026-2029, 2006.

15. Kim J, Takeuchi H, Lam ST, et al: Chemokine receptor CXCR4 expression in colorectal cancer patients increases the risk for recurrence and for poor survival. J Clin Oncol 23: 2744-2753, 2005.

16. Hiller DJ, Li BD and Chu QD: CXCR4 as a predictive marker for locally advanced breast cancer post-neoadjuvant therapy. J Surg Res 166: 14-18, 2011.

17. Xie L, Wei J, Qian X, et al: CXCR4, a potential predictive marker for docetaxel sensitivity in gastric cancer. Anticancer Res 30: 2209-2216, 2010

18. Spoo AC, Lubbert M and Wierda WG: CXCR4 is a prognostic marker in acute myelogenous leukemia. Blood 109: 786-791, 2007.

19. Laverdiere C, Hoang BH and Yang R: Messenger RNA expression levels of CXCR4 correlate with metastatic behavior and outcome in patients with osteosarcoma. Clin Cancer Res 11: 2561-2567, 2005

20. Sun YX, Wang J, Shelburne CE, et al: Expression of CXCR4 and CXCL12 (SDF-1) in human prostate cancers (PCa) in vivo. J Cell Biochem 89: 462-473, 2003.

21. Zagzag D, Krishnamachary B, Yee H, et al: Stromal cell-derived factor-1alpha and CXCR4 expression in hemangioblastoma and clear cell-renal cell carcinoma: von Hippel-Lindau loss-offunction induces expression of a ligand and its receptor. Cancer Res 65: 6178-6188, 2005.

22. Thomas CW, Claudine G, Stefan B, et al: Strong expression of chemokine receptor CXCR4 by renal cell carcinoma correlates with advanced disease. J Oncol 2008: 1-6, 2008.

23. Wang LH, Liu Q, Xu B, et al: Identification of nuclear localization sequence of CXCR4 in renal cell carcinoma by constructing expression plasmids of different deletants. Plasmid 63: 68-72, 2010.

24. Lin SY, Makino K, Xia W, et al: Nuclear localization of EGF receptor and its potential new role as a transcription factor. Nat Cell Biol 3: 802-808, 2001.

25. Sherr CJ: Cancer cell cycles. Science 274: 1672-1677, 1996.

26. Khan MZ, Shimizu S, Patel JP, et al: Regulation of neuronal P53 activity by CXCR 4. Mol Cell Neurosci 30: 58-66, 2005.

27. Gerlach LO, Skerlj RT, Bridger GJ, et al: Molecular interactions of cyclam and bicyclam non-peptide antagonists with the CXCR4 chemokine receptor. J Biol Chem 276: 14153-14160, 2001.

28. Dewan MZ, Ahmed S, Iwasaki Y, et al: Stromal cell-derived factor-1 and CXCR4 receptor interaction in tumor growth and metastasis of breast cancer. Biomed Pharmacother 60: 273-276, 2006. 
29. BurgerM, Glodek A, Hartmann T, et al: Functional expression of CXCR4 (CD184) on small-cell lung cancer cells mediates migration, integrin activation, and adhesion to stromal cells. Oncogene 22: 8093-8101, 2003.

30. Hartmann TN, Burger JA, Glodek A, et al: CXCR4 chemokine receptor and integrin signaling co-operate in mediating adhesion and chemoresistance in small cell lung cancer (SCLC) cells. Oncogene 24: 4462-4471, 2005.

31. Chao DT and Korsmeyer SJ: Bcl-2 family: regulators of cell death. Annu Rev Immunol 16: 395-419, 1998.

32. Carmen HL, Jaris V, Laura Hi, et al: CXCL12/CXCR4 signaling promotes human thymic dendritic cell survival regulating the Bcl-2/Bax ratio. Immunol Lett 120: 72-78, 2008

33. Zheng H, Dai T, Zhou B, et al: SDF-1alpha/CXCR4 decreases endothelial progenitor cells apoptosis under serum deprivation by PI3K/Akt/eNOS pathway. Atherosclerosis 201: 36-42, 2008
34. Gao F, Gao E, Yue T L, et al: Nitric oxide mediates the antiapoptotic effect of insulin in myocardial ischemia-reperfusion: the roles of PI3-kinase, Akt, and endothelial nitric oxide synthase phosphorylation. Circulation 105: 1497-1502, 2002.

35. Yin G, Li LY, Qu M, Luo HB, Wang JZ and Zhou XW: Upregulation of AKT attenuates amyloid- $\beta$-induced cell apoptosis. J Alzheimers Dis 25: 337-345, 2011.

36. New DC, Wu K, Kwok AW, et al: G protein-coupled receptorinduced Akt activity in cellular proliferation and apoptosis. FEBS J 274: 6025-6036, 2007.

37. Ljungberg B, Cowan NC, Hanbury DC, et al: EAU guidelines on renal cell carcinoma: the 2010 update. Eur Urol 58: 401-402, 2010. 